\title{
Treatment Resistant Depression in primary care: co-constructing difficult encounters
}

\begin{abstract}
Many patients with depression do not recover despite medication or therapy. Individuals with Treatment Resistant Depression (TRD) often have co-morbid anxiety, personality difficulties, drug or alcohol misuse and have been characterised as difficult, heartsink or problem personalities by GPs (McPherson \& Armstrong, 2009) yet critical studies of interaction in medical settings suggest that the context may have a role in constructing the patient. Twelve audio-recorded routine consultations were analysed following guidelines for qualitative analysis of medical discourse (Waitzkin, 1990). The interpretation focused on ways in which the context and structure of primary care consultations in a UK setting construct difficult encounters which may lead to patients with TRD being seen as difficult to manage in various ways. Three over-arching observations were that presentation of multiple problems in multiple domains clash with the consultation format; that patients' atypically high level of activity in a timelimited setting prevents patient-centred work; that the question and answer format restricts multifaceted discussions of social and emotional problems, preventing shared understandings emerging. However, although interactions appear uneasy, they are repaired and may be moderately palliative. Suggestions are made for re-orienting GP work with TRD towards long-term goal setting outside of the traditional consultation structure in order to develop shared understandings.
\end{abstract}

Keywords: UK, primary care, General Practitioners, depression, medical discourse, qualitative analysis, doctor patient interaction 


\section{Introduction}

Treatment Resistant Depression (TRD) is associated with high physical and psychiatric comorbidity, poor general functioning and quality of life (Greden, 2001). Patients often have several presenting problems at each consultation which are difficult to untangle and may consist of any combination of physical, social or emotional problems. Although there are no diagnostic criteria for TRD in psychiatric classification manuals, TRD has been defined in the literature as current Major Depressive Disorder (MDD) lasting at least two years and failing to respond to at least two courses of antidepressant medication with varied definitions of what constitutes 'failure to respond' and 'course of antidepressant' (Berlim \& Turecki, 2007). Carrying out a psychiatric assessment of MDD or monitoring responses on depression scales to each course of antidepressant are not routinely carried out in primary care. Nor do these technical measurements reflect primary care encounters where immediate medical problems along with social and functioning issues are often more pertinent than depressive symptomatology.

In the UK, primary care acts as the gateway to services for people with depression. In 2006, Lord Layard instigated a UK policy initiative Increasing Access to Psychological Therapies (IAPT) to reduce the overall economic burden of depression by increasing access to evidence based psychological treatments (particularly Cognitive Behavioural Therapy, CBT) in order to reduce the disability benefit bill (Layard 2006). The subsequent roll-out of IAPT services nation-wide indicated a recognition of the economic need to treat depression. Nevertheless not all treatments are successful, TRD representing past failures of natural resolution and current management strategies. It is more burdensome than other forms of depression (Greden 2001) and there is little 
evidence that it be successfully treated in the long term (McPherson et al. 2005). The burden of care for TRD is likely to remain within primary care where GPs are largely working without TRD-specific evidence based guidance (since most guidance relates to mild and moderate forms of depression).

GPs report that patients presenting with TRD may be associated with the labels 'heartsink' or 'difficult' and that GP loss of empathy and frustration can occur (McPherson \& Armstrong, 2009). The concepts of 'heartsink' and 'difficult' tend to characterise patients from a medical perspective and fail to acknowledge the possibility that patient types may be constructed within medical encounters by the nature of the interaction. For example, Schwenk et al. (1989) argue that differing expectations of patients and GPs can lead to problematic consultations and hence to the GP thinking of the patient as 'difficult', particularly if the GP is unable to derive professional satisfaction from the encounter because of their perception of the patient's style or personality. Similarly, May et al (2004), investigating GP responses to chronic problems such as depression, back pain, menhorrhagia and medically unexplained symptoms, emphasise that the negotiation of patients' chronic problems takes place within a highly complex frame and that social influences on the primary care encounter have an unavoidable influence on the doctor-patient interaction. Also concerning medically unexplained symptoms, Salmon (2007) has shown that primary care consultations often involve contest between patients' expertise regarding their own experience of symptoms and GPs' expertise based on medical tests and that both parties may use different strategies to impose their authority on the consultation. GPs can inadvertently cause the patient to exacerbate their somatisation of any psychosocial 
difficulties by legitimizing the complaints with diagnoses and tests whilst ignoring psychosocial cues (Salmon, 2006).

The present study is concerned with how patients with TRD and GPs co-construct difficult consultations in context of the primary care setting. We analyse interactions between GPs and patients in cases where the patients have depression which has not responded to previous treatment attempts. While GPs may construe these interactions in relation to a feeling of 'heartsink' or as caused by disordered personalities of patients (McPherson \& Armstrong, 2009), the present analysis focuses on ways in which difficult interactions may arise from the medical context which imposes constraints on the number and nature of problems a patient may present in a single consultation.

\section{Methods}

That the context may shape the nature of an interaction rather than the patient's character or their condition has been proposed by various authors and examined using variations on observational methods. Strong (1979) employed a systematic observational method to demonstrate the ways in which political, social and economic factors along with an intrinsic medical dominance can shape doctor-patient interactions. He analysed 'role formats' employed by participants and showed the ways in which these were constrained by social contexts and were institutionalised. Similarly, Waitzkin (1989) argued that medical encounters contained ideologic messages and mechanisms for social control, albeit unconscious on the part of the professional. He developed a form of critical interactional analysis which sought to identify the exercise of power and authority by physicians in medical encounters. Waitzkin's method has 
been criticised by conversation analysts for its top-down approach to identifying deficiencies in physician behaviour and assuming capitalism and paternalism to be the inherent cause (Schlegoff, 1991). However, Waitzkin (1990) notes conversely that conversation analysis tends to ignore problems such as selective bias by adopting a narrow focus on patterns in language, excluding context and avoiding interpretation. Similarly, critical discursive psychologists argue that it is both useful and appropriate to attempt to make psychological interpretations (as well as cultural and linguistic interpretations) in analyses of language produced in social contexts (Wetherell, 2007). The analytic method employed here follows Waitzkin's (1990) criteria which include that the sampling of discourse should be clearly described, that the analysis and interpretation should address both content and structure of the texts and that the reliability of the analysis and interpretation should be assessed by multiple observers.

\section{Data source}

The cases for the present study were selected from amongst 506 routine consultations from five GP practices in London from May 2004 to February 2005. The present study is a sub-study within this larger study which was concerned with the interactions between patients and doctors and how depression is managed in general practice (see also Karasz et al, 2012). Data collection took place around the time the Quality Outcomes Framework (QOF) was introduced in the UK to incentivise GPs around various indicators of patient care, but prior to the introduction of depression indicators. Data collected included Hospital Anxiety and Depression questionnaires (HADS) (Zigmond \& Snaith, 1983); a patient questionnaire about the patient's history of depression; a GP questionnaire about the patient; and audio-recordings of the 
consultation. A favourable ethical opinion was given for both the larger study and the present sub-study by Lewisham Research Ethics Committee.

\section{Selecting cases}

Waitzkin (1990) notes the importance of employing a clear systematic sampling procedure for selecting examples of medical discourse for analysis. In the present study, the aim was to identify cases for whom TRD might be a reasonable working label. Given the limits of the data available, our criteria only allowed for us to look at one year duration rather than two. All cases that met the following criteria were included:

1. HADS depression score of 11 or more out of 21 , recommended as a cut-off for caseness (Zigmond \& Snaith, 1983)

2. Patient reported having depression for more than a year or having been depressed in the past

3. Patient reported having used antidepressants in the past or having found antidepressants unhelpful

4. The consultation included discussion of emotional problems.

Twelve of the 506 consultations met the inclusion criteria. Table 1 summarises the general features of consultations.

\section{Coding and exploring data}

Consultations included in the study were transcribed by an independent transcriber, using standard conventions (Heritage, 2004). Qualitative analysis software was used. Following Waitzkin, analysis involved observation of both structure and content. To 
examine structure, each segment of text was coded according to the observable sub-goal (opening the conversation, identifying the problem, exploring the problem, discussion or decision on how to address the problem and closure). Subsequently, each participant's turn at talk was coded thematically according to its content, taking both the topic and action into account. Interruptions and pauses employed by each participant to keep or hand over their turn (interpreted according to the outcome i.e. turn continued or turn lost) were observed and coded in order to give a sense of how active or passive GPs and patients were in constructing the encounter. Codes and themes were generated by a bottom-up process of analysis rather than generated a-priori; as new codes emerged during the analysis, previous transcripts were checked for consistency in use of codes. On completion of an initial analysis by $\left[1^{\text {st }}\right.$ author], samples of transcripts were reviewed by $\left[2^{\text {nd }}\right.$ and $3^{\text {rd }}$ author] against the final coding frame. There were very few instances of disagreement and these were generally resolved following discussion and clarification. Interpretation was carried out collaboratively by all authors and consisted of a synthetic analysis of themes and codes (structure and content). Three extracts are provided along with individual quotes where relevant. Full transcripts are available on request.

\section{Analysis}

Interpretation led to three broad observations concerning the ways in which consultations for patients with TRD may be constructed as difficult by the context of the primary care encounter rather than solely by the GP or patient in response to the other's behaviour or personality. These were first, that the mutually understood structure of a GP consultation is transgressed by the presentation of multiple problems in multiple domains (physical, emotional, social, functional) and of both acute and 
chronic type. Second, that the more active and assertive behaviour of the patient, than is typical in primary care, does not lead to integrated whole person care. Third, that the mutually understood structure and format of consultations based around disease models with standardised questions restricts multifaceted discussions of social and emotional problems. Detailed analyses leading to this interpretation follow.

\section{Multiple problems}

In a UK based CA study of 237 consecutive consultations, Campion and Langdon (2004) observed that in a third of consultations, patients discuss more than one topic. In the present study, all patients introduced several problems and these were in more than one domain (physical, emotional, social or functional). Yet, problems were not all identified at the outset of the consultation. In response to varied opening questions, patients either gave vague brief responses (describing a brief single problem, yet going on to bring up other problems at later points in the consultation) or they gave lengthy complex answers. The introduction of new problems by patients spanned the whole length of the consultations.

Because patients and GPs both introduced problems for discussion at various points, the actual sequence of events tended to be very complex, moving back and forward through the stages until a final closure could be reached. Unsurprisingly then, the length of consultations (see Table 1) were often greater than sample GP norms.

Patients used a range of opportunities and styles to introduce new problems. Patients' direct questions, for example, often contained hints about psychosocial problems already mentioned but that the GP had not followed up. They usually failed, however, 
to shift the GP's attention beyond a response to the explicit question and the GPs tended to continue with the activity of prescribing or typing. Direct questions were often in relation to medication being prescribed and were invariably asked at times when the GP was writing a prescription or typing.

\section{Extract 1: C11}

1. GP: okay (.) how $\downarrow \underline{\text { are }}$ things?

2. PT: mm (.) I dunno if you've [seen on there (.) (bout) the crash umm:

3. GP: [umm you had an accident (.) $\uparrow$ yeah?

4. PT: pain's gone alright $\{$ keyboard noise $\}{ }^{\circ}($ still like $)$ shit $^{\circ}$ \{keyboard noise?\}

5. GP: feel very anxious don’t you \{keyboard noise $\}$ what $\uparrow$ happened? (.2)

15. PT: =erm (.) headaches haven't gone (.1) \{keyboard sounds continue while PT

16. talks $\}$ it's gone $\underline{\downarrow \text { today }}$ actually (.) but yesterday $\mathrm{I}$ had $=\mathrm{a}=$ heada:che a:ll day

17. .hh ( ) Doctor (Tan) gave me (.) is it $\uparrow$ Diazepam?

18. GP: $\uparrow$ yeah?

19. PT: they=er helped me (.) sleep but only (.) initially and (.) sleeping at nights w'I

20. just .hh: I'm jus' not sleeping (.2) I sleep mo:re during the day (.3) \{keyboard

21. stops, then intermittent under following talk\}

22. GP: (see it's) the shock is coming through $\downarrow_{\text {now isn't it? }=}$

23. PT: $=$ possibly $=$

31. GP: it is (.) yeah it does (.) make you very anxious and shaky=

32. PT: =umm (.) I dunno if it's (.) I dunno if it's where I can:'t (.) get comfortable

33. of a ni:ght (.1) but the back (.) top of my back ( I now) get backache (.) but I 
34. dunno if that's just where I can:'t (.) get comfortable

137. GP: and try these one (.2) one every night=

138. PT: $=$ okay $(.2)>$ will that $<$ help me sleep as well?=

139. GP: =yeah $($.$) that is wise=$

140. PT: shall I carry on taking the Diazepam? (.)

141. GP: erm (.) I think $\downarrow$ stop the Diazepam (.) (noises keyboard?) it's it's more of

142. a (.) anti ( $\quad$ ) drug tha:n (.1) er it does help the muscle [(.) relax

143. PT: [yeah relax (.) relaxing=

In C11, the GP introduced a new medication indicating that it would "relax" her, should be taken at night and that a "hangover" feeling the next day may require "sleeping off" (lines 75-80, not quoted in full). In Extract 1, the patient asks later on of the new medicine, "will that help me sleep as well?" (line 138). Earlier in the consultation the patient had repeatedly noted a concern with sleep (lines 19-20 and 32-34), following a concern about headaches, but the GP never explicitly discussed sleep problems; instead the GP briefly infers that all the symptoms are related to the crash. Perhaps understandably then, the patient still seems unsure whether the sleep issue is being dealt with by the new prescription.

The exchanges suggest that patient and GP may have different beliefs about the aetiology of the headache and sleep disturbance (pain as a physical discomfort versus 'shock' as a psychological discomfort, see lines 5, 22, 31). The patient's question may result from a lack of confidence in the GP's understanding and a failure of either to 
discuss a multifaceted link between pain, 'shock', anxiety and sleep, constituting in a relatively short consultation (6.47 minutes).

Similarly, in C8, the patient makes reference to drinking a bottle of wine a day couched within a discussion about sleep and pain. The alcohol issue was not taken up, but the patient makes the following comment later on while the GP is preparing a prescription:

PT: otherwise who knows erm .hh but also can I check back with you (.) umm the codeine I'm on can I take it three times a day or only twice a day? \{noise cos that along with wine and cigarettes gives me instant $\{$ keyboard noises .3$\}$ this is ridiculous what am I doing $?=(\mathrm{C} 8$, line 77$)$

In this turn, the patient notes for the second time in the consultation her practice of combining alcohol and painkillers, but the GP does not follow it up either time and completes the prescription. The doctor listens for several minutes to the patient's complicated and not insignificant social issues indicating willingness to use time for listening but not to engage in a more complex task of working the issues into a formulation and disposal.

GPs did nevertheless introduce and discuss psychosocial issues during the consultations using both exploratory and interrogate questions indicating a shared understanding that the patients had existing social and psychological problems. However, GPs tended not to follow-up specific psychosocial issues when brought up by patients (as described in C8 above) nor the specific causal explanations patients put forward. For example, in C4 (see Extract 2), the patient begins by describing problems following a recent 
hospital admission. The GP is the first to make reference to the patient's known depression (line 77).

\section{Extract 2 (C4)}

75. GP: can we check it now your blood pressure?

76. PT: please \{sounds of taking blood pressure

77. GP: have you run out of Venlafaxine as well (.) for your depression?

78. PT: is it ( )Amalo blood ( )

79. GP: Amlodipine is the blood pressure tablet

80. PT: yeah that's the one [erm

81. GP: [and (Venlafaxine is the depression one

82. PT: ( $)$ \{blood pressure being taken $\}$

83. GP: is the depression the same?

84. PT: ${ }^{\circ}$ umm what do you think it's about the same ${ }^{\circ}$

85. GP: $\{$ noises $\}$ umm

86. PT: it doesn't get worse

87. GP: are you better off it or on it? (.) or does it vary?

88. PT: it varies $\{$ noises $\}$

89. GP: umm (.) are you currently coping without it?

90. PT: no not really because \{pumping sounds

91. GP: you need some more then

92. PT: I've got no one to ((laughs)) I'm alw(h)ays on my own ?: ((laughs)

93. PT: so(h)rry hh .hh oh (.) hh oh (.7) hh \{ sound of air release $\}$

94. GP: oh your blood pressure is alright today 
115. PT: yes ${ }^{\circ}$ yes I think I'm going back on depression tablets again ${ }^{\circ}$

116. because really there was some you know was honestly I wanted to die

117. ( ) just everything was (.) around me you know

118. GP: $\mathrm{mm}$

119. PT: ( ) the children everything which was (.) .hh (.)

120. so I'm saying it doesn't get worse doesn't get better

121. GP: okay but I think without (Venlafaxine) you feel worse

122. PT: $\uparrow(\quad)$ oh before I was taking it originally ( ) I I was in the

123. same mood I was in the same thing .hh hh I've got nobody got no

124. friends

125. GP: $\mathrm{mm}$

126. PT: any little (.) th thing comes between er between ( ) puts me

127. off and I don't want to know them (.) I end up on my own

290. GP: there you go my friend

291. PT: sorry doctor hh f fast typing $\}$ you must have a lot of patience (.)

292. with yourself you know you've gotta help me you've gotta listen to

293. every(h)body when is crying on your shoulder every(h)time

294. GP: it's okay

295. PT: oh God ((laughing))

296. GP: that's what we're here for

297. PT: obvious(h)ly ((laughs)) oh I feel better when I see y(h)ou

298. $\quad$ GP: good 
In lines 83-94, the GP draws on a model of medication efficacy linked to disease worsening or improvement whilst the patient indicates (line 92) that she attributes her depression to loneliness, implying both that she would like to discuss this and that this negates her needing more Venlafaxine (although she also agrees that she needs more, indicating some ambivalence). Following a further discussion of blood pressure medication, the GP reiterates (line 121) the need for medication relating to feeling 'worse' to which the patient again sets up loneliness in contradiction (lines 122-127).

The GP acknowledges the patient's distress and allows her to talk, but does not actively take up or build on the patient's way of conceiving her problems. Instead he reminds the patient that he referred her to therapy before, at which point she takes up another tack, relaying concerns about her memory. He eventually closes down the patient's talk by giving her the Venlafaxine prescription without having addressed her implicit suggestion that it will not help her loneliness; he also agrees to a referral to the memory clinic without formally assessing memory or explaining how this could be a symptom of depression. In this way, the GP appears to allow psychosocial explanations and problems to be discussed, but only those which fit within the "detect and treat model" of depression care are dealt with (i.e. the depression has not improved so a new prescription is issued). The GP does not fully enter the patient's world and neither does he help co-construct a psychiatric formulation which includes 'loneliness' and the patient's accounts of why her friendships break down. This lack of ease between doctors' and patients' frameworks contrasts with doctors' willingness to follow patients' leads in more straightforward cases (Karasz et al 2012). This might represent a lack of time or skill to engage in complex issues or a belief that these issues are not 'doctorable' beyond listening. 
GPs and patients appear to have differing priorities in all consultations examined. In C3, for example, the patient identifies his problem as drinking too much, but tacitly weaves in links to low self-esteem, relationship problems, work, lifestyle and panic attacks while the GP follows an alcohol dependence model of enquiry and referral. Similarly in C9, the patient links his alcohol use to anxiety, depression and paranoia about doctors while the GP separates these arguing that he cannot treat the patient's depression when he is drinking. In this case, the doctor explicitly indicates that the patient is undeserving of treatment because of his drinking (see Extract 3 lines 18-19).

\section{Asymmetry in GP-Patient Interaction}

Asymmetry refers to doctors asking most of the questions, interrupting the patient, deciding what is relevant and generally imposing more authority on the interaction than the patient (Pilnick \& Dingwall, 2011). Robinson (2003), analysing routine primary care consultations, observed that "patients rarely ask for information, explanations, or clarification, or volunteer information, opinions, preferences, or concerns." Similar findings permeate studies of doctor-patient interaction (Pilnick \& Dingwall, 2011). Patients in this sample not only contributed lengthy descriptions of their problems but also frequently interrupted the GP, rejected advice, defended themselves against criticism, noted inadequate health care services or treatments they had received, asked direct questions and occasionally even criticised the GP. For example, patients interrupted the GP on average 14 times per consultation (range 1-33), a similar rate to GPs who interrupted patients 15 times on average (range 1-36). Patients challenged, contradicted or rejected the GP's advice on average 3 times per consultation (range 011) again similar to GPs contradicting, challenging or refusing the patient's request (3 
times on average, range 0-10). On these criteria, at least,patients and GPs in this sample appear equally matched in terms of imposing authority.

Nevertheless, patients demonstrated an understanding that long accounts of their problems were not necessarily permitted within the context of a GP consultation by preparing the GP in advance for a long turn, for example:

PT: oh gosh (.) umm (.) ( ) this could be a while um this could take a whi(h)le (C5, line 5)

Patients also used various speech strategies to prevent interruption. These included pausing for breath mid-sentence rather that at the end of a sentence, making words at the end of an utterance run on into the next utterance, not pausing at the natural end of an utterance and interrupting. Patients used these techniques much more often than GPs, particularly in problem identification and problem exploration stages of the consultation and most often when describing social problems suggesting these were perceived to be the most illegitimate topics of conversation.

Given that patients with TRD are by definition a group with chronic experiences of health services and little experience of treatment success, it is perhaps unsurprising that those choosing to continue to seek medical help demonstrate qualities of persistence seen for example in repeated themes (e.g. sleep in C11, loneliness in C4). Within a patient-centred doctrine, this type of encounter might be the ideal opportunity for GPs to engage with the non-passive patient and explore the patient's perspective. Yet their familiarity with the patient and their history along with the immediate context of a full 
waiting room may instead lead the GP to attempt to limit or close down these lengthy narratives (May et al, 2004; McPherson \& Armstrong, 2012). Rather than engage with the patient's narrative, GPs also employed strategies for holding on to their turn to prevent interruptions. Typing, printing, writing and changing the subject all appeared to correlate with non-engagement with patient narratives. For example, in Extract 2 lines 83-94 the GP interrupts the patient (line 91), measures blood pressure throughout the exchange and changes the subject (line 94) to announce the result of the blood pressure test, all resulting in the patient's loneliness being treated as small talk. Rather than patient non-passivity leading to an overall patient-centred approach therefore, it appears to generate both autocratic responses and care which appears reactive to individual symptoms.

\section{Standardised Questions}

The expectation of an ongoing question and answer format for the exploration of problems appears to create further difficulties for patients wishing to discuss various social and emotional problems. This is perhaps most evident when observing what appear to be standardised questions.

Boyd (2006) observed that standardised questions forced by protocol tend to be coconstructed for no-problem, brief responses. There are three main areas in which standardised questions emerge in the present data set: suicide risk, social support and alcohol use. Notably there is no evidence of depression checklist questions, perhaps because depression is already an established diagnosis or because use of such questions to monitor level of depression is not established practice. 
The box below shows all instances in which GPs asked about suicide risk.

\begin{tabular}{|l|l|l|}
\hline C2 & okay .hh do you feel life's worth & Patient \\
living? & oh yeah ah yeah yeah I mean .hh I've had \\
this er (.) since nineteen ninety er then .hh \\
I've had no problems with it [no
\end{tabular}

The suicide risk questions above are, with one exception, phrased in a closed format requiring a 'yes' or 'no' response suggesting that they are constructed by the GP for a brief response. But brief responses are not forthcoming and all patients give relatively long responses indicating potential problems. The exception is $\mathrm{C} 4$ in which the GP uses a rhetorical format and adapts it for the individual context which effectively strengthens the possibility of a no-problem response by offering a moral reason for the 
patient to value life. As seen in Extract 2 which also comes from C4, the GP and patient had repeatedly switched between discussion of blood pressure and depression. The GP had restricted the discussion of depression to prescribing Venlafaxine while the patient had attempted to re-formulate her depression as loneliness. The suicide risk question detailed in the box above followed immediately on from Extract 2. The GP's reference to 'valuing life' is assumed to relate to the start of the consultation in which it is evident that the patient had recently had a hospital admission for myocardial infarction. By constructing the rhetorical question in this way, the GP seems to reorientate the discussion from the emotional while also achieving a basic risk assessment and imposing a 'no-problem' response. The patient does not comply and goes on to talk more about her loneliness and implies that she does not feel life is worth living. The GP moves on as though the risk assessment were complete. Indeed all patients except C2 indicate some degree of suicidal thoughts, even if their comment only implies it. None of the GPs follow up these comments. For example, in C9 after the patient hints at suicidal thoughts, the GP continues typing and moves on to the next question "are you under any sort of financial pressure?". This indicates again that GPs seem to want to close down, perhaps out of a sense of hopelessness, a knowledge that suicidal thoughts have been ongoing, may be time consuming to discuss and may be unlikely to benefit from referral to psychiatric services in the absence of a more explicit and immediate suicide threat.

The exception in patient responses to suicide questions is $\mathrm{C} 2$ in which the patient's response seems unrelated to the question. This patient initially presented his symptoms as not eating and diarrhoea, hinted at a connection with a recent stay in a respite centre, 
but clearly marked out his HIV status as an unnecessary line of enquiry. The GP then followed a physical line of enquiry until the patient comments:

PT: it's just ( $\quad$ ) you know I I feel sort of (.) \{noise $\}$ down and you know because .hh (everything's) working okay and my (medication) and all that $(\mathrm{C} 2$, line 32$)$

In this comment, the patient hints at a psychosocial problem but repeats the stress on it being unrelated to HIV. The GP then follows a line of discussion which reveals the patient's ongoing problems with neighbours combined with his disappointment that the respite was in a different place to usual where he did not feel he was looked after well and had returned feeling more depressed than before. Therefore, the patient's response to the suicide risk question may in fact highlight his concern that the GP is making assumptions about HIV patients feeling suicidal, rather than focusing on his living situation. The GP appears not to understand the patient's response and rephrases the question: "d'you feel at all suicidal?" to which the patient responds "no no no". The GP remarks "excellent" and moves on to problem disposal. It therefore appears that while the risk appraisal may have established that the patient does not feel suicidal as a result of his HIV it may be misleading in terms of overall suicide risk.

In contrast, enquiries about alcohol and social support are followed up more consistently, although only three GPs ask a social support question as shown below. 


\begin{tabular}{|c|c|c|}
\hline & GP & Patient \\
\hline C6 & $\begin{array}{l}\text { have you got support? } \\
\text { \{noise }\}\end{array}$ & $\begin{array}{l}\text { mmm yeah you }\{\text { noise continues }\} \text { [GP: } \mathrm{mmm}] \text { you } \\
\text { I don't really ( ) talk talk to noone else Doc (.) I } \\
\text { mean people come to me lot of the family come to } \\
\text { me with their stress when they got problems= }\end{array}$ \\
\hline $\mathrm{C7}$ & $\begin{array}{l}\text { have you got a support } \\
\text { yourself? are you-? (.1) } \\
\text { have you got a partner? }\end{array}$ & $\begin{array}{l}\text { ((sniff)) ummm it's not good 's not good re'(.) } \\
\text { relationship }\end{array}$ \\
\hline $\mathrm{C} 8$ & $\begin{array}{l}\text { okay but have you got } \\
\text { other support though? [P8 } \\
\text { erm:] other colleagues? }\end{array}$ & I've got a clea:ner \\
\hline
\end{tabular}

All of these responses are followed up by GPs more than once. In C6 the GP follows up with “why's that?” and later, “you keep it all within yourself din't you?”. In C8, the patient goes on to give a lengthy account of her friendship with her cleaner and why she values it, all the while encouraged by the GP. These lengthy responses and follow-up probes and questions by GPs suggest that the standard 'social support' question is not co-constructed as brief or requiring a no-problem response, indeed both participants appear comfortable in elaborating and pursuing the discussion.

GP questions on alcohol were found to be numerative and patient responses biographical, as observed by Boyd (2006). In C3, the patient presents his visit primarily as a 'confession' of his alcohol problem. The GP plays relatively little part in this, hence after a lengthy problem identification, the GP asks “=yeah erm (.) how bad?", to 
which the patient gives an even more lengthy reply describing his lifestyle, running a bar, his current and previous relationships, providing an essentially biographical rather than numerical response. The GP eventually is able to rephrase his first question asking, "in terms of units of alcohol (.) how many a week (.) do you reckon?" to which the patient gives detailed accounts of his daily routines and social variables that affect his drinking habits. In spite of the patient's lengthy and detailed formulations of his problem as a psychological issue and his reference to previous depression, the GP is guided by a quantitative alcohol dependence paradigm, avoiding the complexity of interactions between mental health and substance misuse. This has implications for the eventual diagnosis and referral to an alcohol service.

In $\mathrm{C} 9$ and $\mathrm{C} 12$ there is evidence that both alcohol and drug use are expected behaviours in the medical context. After the patients have responded to the standard 'how much?' question, the GP pauses or repeats the patient's response questioningly. In both cases, the patient reiterates their denial showing awareness that the GP may be doubting their response. In C9 this issue becomes yet more apparent as the GP catches the patient out (see Extract 3).

\section{Extract 3: C9}

10. GP: un hunh \{typing sounds .1$\}$ right how much are you drinking at the moment?

11. $\{$ keyboard click $\}(.1)$

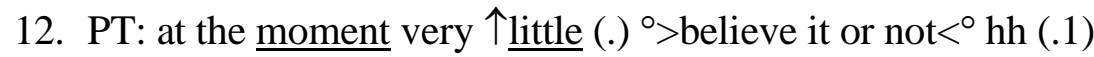

13. GP: what is mean $>$ what do you mean $<$ very little? $=$

14. PT: =very very little $>$ I only drink one $(\quad)$ indoors $<$ and (there's) $(.1)$ \{noise

15. 'll be once: once a week (.) maybe once a $\uparrow \underline{\text { fortnight }}(.1)$ 
16. GP: un hunh (.2) and you are drunk again $\downarrow$ today? (.1)

17. PT: I: just had $\mathrm{a}^{\circ}(\mathrm{few})^{\circ}=$

18. GP: =um (.1) knowing that you are coming to see a doctor: (.1) and you want 19. treatment for depression and then you (.1) drunk

This consultation results in the patient and GP orientating to very different understandings about the patient's difficulties and the patient then becomes so distressed that the GP is unable to end the consultation effectively and arranges for the patient to return the following morning in order to make a referral to the mental health team. It is only at this point that the GP's previously separatist stance on alcohol and mental health breaks down: stating that the mental health team will "approach your problem holistically" and work with drug and alcohol services to "sort out your problem”. Perhaps this was possible in the knowledge that the problem was being handed on to a service with increased consultation times enabling holistic assessment.

While the present data set indicate that alcohol is co-constructed as a taboo issue in that C12 emphasises he is not a 'violent drunk' and C9 is chastised for drinking before his appointment, it is also evident that there is a medical model for identifying and managing 'alcoholism' which is treated by GPs as distinct from the 'depression' model and this can lead to parts of patients' presentations being ignored. It is also notable that GPs only raise alcohol issues with men and that even when one female patient referred to her overuse of alcohol ( $\mathrm{C} 8$ described above), her comments were not picked up by the GP who stayed working from within a depression model. 


\section{Discussion}

While primary care interactions have been examined by others in relation to depression (e.g. May, 2004) and other long term conditions (e.g. Salmon, 2007), this is the first study to examine in detail consultations in which patients have chronic, complex depression that has not responded to antidepressants. Whilst many common features of primary care encounters are evident here, the study highlights some specific issues for this group of patients which are particularly important given the lack of evidence based secondary care treatments available (McPherson et al, 2005).This paper has highlighted how the medical paradigm can impose specific limits on shifts into and out of non-medical discourse by patients and GPs, particularly when patients have multiple problems in different domains (physical, emotional, social), leading to problems being managed in disaggregated ways. Secondly, we have shown that the behaviour of the patient as less passive and compliant than is typical does not improve the balance or symmetry of authority and also does not result in more whole-person care. This suggests that 'asymmetry' is more complex than an imbalance in authority and that the imbalance cannot simply be addressed by patients increasing their use of authority. Thirdly, we have suggested that the mutually understood structure and format of consultations based around disease models with standardised questions restricts multifaceted discussions of social and emotional problems.

These observations might be considered likely to lead to encounters between GPs and patients with TRD being experienced as difficult; to the patient being constructed as difficult by the GP; and potentially also to the GP being constructed as unhelpful by the patient. It is important to acknowledge however, that these observations are based on a 
single consultation per patient which may not be representative of the ongoing relationship between these likely frequent attenders and the GP. Moreover, we can only speculate on how these particular participants may have experienced the encounter and how the encounter may have contributed to their overall perception of the other party. It may have been useful to have interviewed GPs and patients after the encounter, possibly including Taped-Assisted-Recall methods to enhance the findings and it is important to note that GP practice may have altered in the years since the data was collected. Nevertheless, the detailed observations made in the present study can usefully be considered within the context of other studies cited previously which indicate that patients with TRD can be constructed as difficult by GPs.

It is also possible that in spite of the difficulties inherent in these encounters, of never reaching an integrated formulation, shared understanding or a way forward, the visit may have some palliative effect on these patients (see for example, Extract 2, lines 290298). Pare (1999) provides a range of advice to GPs managing difficult patients which includes realising that "tolerating" patients may be the best treatment for them; the GPpatient relationship offers a rare opportunity for patients to have a regular positive interpersonal contact, the importance of which can be seen in some of the endings in this data set in which relationship repairs are constructed and then expressed as exaggerated expressions of farewell during the closing segment (as in Extract 2, line 290). These repairs may be particularly important because of the mutual knowledge that the relationship is a long-term one and that future encounters are inevitable within a relatively short space of time. 
It is important to consider whether there are any ways in which the present interpretation of contextual barriers may lend itself to suggestions for improving GP care for this complex group of patients, particularly given the opportunity offered by a relatively stable relationship to patients who may normally struggle with relational issues. We have suggested that the context (including structure and format) of the GP consultation restricts the ways in which GPs can contribute to effective solutions for this group and that the conversations witnessed suggest recurrent and not always productive discourses. Yet GPs have been shown elsewhere to have working models that do incorporate the social as well as psychological origins of depression (McPherson \& Armstrong, 2012) and we have seen that both parties seem willing to discuss the social and emotional to some extent. In the absence of clear evidence for effective manoeuvres to enhance these discussions, we suggest some approaches.

Firstly, GPs are in a position to work with patients to construct a bio-psycho-social model to explain distress and unhelpful behaviours. Most people with TRD have at least one other diagnosis and many have symptoms that are subthreshold with respect to anxiety, post traumatic stress disorder, obsessive compulsive disorder and personality disorder. These psychiatric "disorders" can be framed as resulting from common social origins such as trauma, abandonment and other ongoing life events, rather than always being seen as separate diseases. This can be done by just alluding to past traumas, or making time to ask about an individual's past, for those who want to talk; it might both catalyse a more trusting relationship and help co-construct an integrated explanatory narrative. Furthermore, self-harm, substance misuse and to some extent eating disorders can be formulated as maladaptive coping responses; these in turn can be contrasted with a focus on an individual's strengths and capacities and perhaps most importantly on their personal goals in the social domains of work, education, housing and relationships. 
Clearly time remains a limiting factor, but when patients present with such chronic complex histories it may be appropriate to circumvent much of the traditional structure and format of consultations in order to make better use of time and to conceive of each individual consultation as one part of an ongoing conversation spanning over multiple meetings, with the aim of achieving specific social goals. GPs are increasingly aware of their role in interpreting evidence for individual patients and Interpretative Medicine (Reeve, 2011) explicitly sees healthcare as a tool for living a better life rather than an end in itself. Giving primacy to the patient's social goals may help contain and focus a series of interactions and even avoid a descent into 'narratives of despair' (Palmer, 2007).

There is also a need for a range of techniques for use with complex cases in primary care settings to help patients make sense of their past and current lives, as well as to develop integrated bio-psycho-social formulations . Clinical supervision is not established in general practice; peer led groups one to one peer support or supervision from a trained therapist, are informal arrangements which are not widespread but could contribute to substantially supporting practitioners to deal with some of the most complex presentations in the NHS.

\section{References}

Berlim MT \& Tureki G (2007) What is the meaning of treatment resistant/refractory major depression (TRD)? A systematic review of current randomized trials. European Neuropsychopharmacology, 17(11):696-707. 
Boyd E \& Heritage J (2006) Taking the history: questioning during comprehensive history-taking. In: Heritage J \& Maynard D (eds) Communication in Medical Care: Interaction Between Primary Care Physicians and Patients (Studies in Interactional Sociolinguistics). Cambridge: Cambridge University Press, pp. 151-184.

Campion P \& Langdon M (2004) Achieving multiple topic shifts in primary care medical consultations: a conversation analysis study in UK general practice. Sociology of Health \& Illness. 26(1):81-101.

Greden J (2001) The Burden of Disease for treatment-Resistant Depression. Journal of Clinical Psychiatry, 62(Suppl 16):26-31.

Heritage J (2004) Conversation Analysis and Institutional Talk. In: Silverman D (ed) Qualitative Research: Theory, Method and Practice, Second Edition. London: Sage Publications.

Karasz A, Dowrick C, Byng R, Buszewicz M, Ferri L, Olde Hartman C, van Dulmen S, van Weel-Baumgarten E, Reeve J (2012) What we talk about when we talk about depression: doctor-patient conversations and treatment decision outcomes. British Journal of General Practice, 62:30-31.

Layard R (2006) The Case for Psychological Treatment Centres. London: Centre for Economic Performance, London School of Economics. 
May C, Allison G, Chapple A, Chew-Graham C, Dixon C, Gask L, Graham R, Rogers A, \& Roland M. (2004) Framing the doctor-patient relationship in chronic illness: a comparative study of general practitioners' accounts. Sociology of Health \& Illness, 26(2):135-58.

McPherson S, Cairns P, Carlyle J, Shapiro DA, Richardson P \& Taylor D (2005). The effectiveness of psychological treatments for treatment-resistant depression: a systematic review. Acta Psychiatrica Scandinavika, 111(5):331-340.

McPherson S \& Armstrong D (2009) Negotiating Depression Labels in Primary Care. Social Science and Medicine, 69(8):1137-1143.

McPherson S \& Armstrong D (2012), General Practitioner Management of Depression: a systematic review, Qualitative Health Research, 22(8):1150-1159.

Palmer V (2007) Narrative repair: (Re)covery, Vulnerability, Service, and Suffering. Illness, Crisis \& Loss, 15(4):371-388.

Pare MF Rosenbluth M (1999) Personality Disorders in Primary Care. Clinics in Office Practice, 26(2):243-278.

Pilnick A \& Dingwall R (2011) On the remarkable persistence of asymmetry in doctor/patient interaction: A critical review. Social Science \& Medicine 72:1374-1382. 
Reeve J (2010) Interpretive Medicine. Supporting generalism in a changing primary care world. Royal College of General Practitioners Occasional Paper Series, 88.

Robinson JD (2003) An interactional structure of medical activities during acute visits and its implications for patients' participation. Health Communication, 15(1):27-57.

Salmon P (2006) The Potentially Somatizing Effect of Clinical Consultation. Primary Psychiatry, 11(3):190-200.

Salmon P (2007) Conflict, collusion or collaboration in consultations about medically unexplained symptoms: The need for a curriculum of medical explanation, Patient Education and Counseling, 67(3):246-254.

Schegloff E (1991) Reflections on talk and social structure. In: Boden D \& Zimmerman D (eds) Talk and social structure: Studies in ethnomethodology and conversation analysis. Cambridge: Polity Press, pp. 44-70.

Schwenk TL, Marquez JT, Lefever RD, Cohen M (1989) Physician and patient determinants of difficult physician-patient relationships. Journal of Family Practice, 28:59-63.

Strong P (1979) The ceremonial order of the clinic: patients, doctors and medical bureaucracies. London: Routledge and Kegan Paul. 
Waitzkin H (1989) A Critical Theory of Medical Discourse: Ideology, Social Control, and the Processing of Social Context in Medical Encounters. Journal of Health and Social Behavior, 30:220-239.

Waitzkin H (1990) On studying the discourse of medical encounters: a critique of qualitative and quantitative methods and a proposal for reasonable compromise. Medical Care, 28(6):473-488.

Wetherell M (2007) A step too far: Discursive psychology, linguistic ethnography and questions of identity. Journal of Sociolinguistics, 11(5):661-681.

Zigmond AS \& Snaith RP (1983) The Hospital Anxiety and Depression Scale. Acta Psychiatrica Scandinavika, 67(6):361-370. 
Table 1: Features of Consultations

\begin{tabular}{|l|l|l|l|l|l|l|}
\hline Consultation & Gender & Gender & Duration & HAD & HAD & Age \\
\hline C1 & Patient & GP & (Minutes) & Depression & Anxiety & \\
\hline C2 & Female & Male & 9.21 & 11 & 16 & 27 \\
\hline C3 & Male & Male & 15.19 & 15 & 17 & 55 \\
\hline C4 & Male & Male & 14.15 & 11 & 18 & 30 \\
\hline C5 & Female & Male & 16.2 & 18 & 16 & 53 \\
\hline C6 & Female & Male & 17.04 & 12 & 9 & 28 \\
\hline C7 & Male & Male & 13.19 & 11 & 7 & 44 \\
\hline C8 & Female & Male & 18.06 & 16 & 18 & 42 \\
\hline C9 & Female & Male & 12.19 & 12 & 14 & 58 \\
\hline C10 & Male & Male & 13.29 & 17 & 16 & 38 \\
\hline C11 & Female & Female & 10.28 & 11 & 16 & 51 \\
\hline C12 & Female & Female & 6.47 & 13 & 11 & 23 \\
\hline
\end{tabular}

